\title{
Influence of round-off errors on the reliability of numerical simulations of chaotic dynamic systems
}

\author{
Shijie Qin ${ }^{1}$ and Shijun Liao ${ }^{1,2}$ \\ ${ }^{1}$ School of Naval Architecture, Ocean and Civil Engineering, \\ Shanghai Jiaotong University, Shanghai 200240, China \\ ${ }^{2}$ Ministry-of-Education Key Lab for Scientific and Engineering Computing, \\ Shanghai 200240, China
}

\begin{abstract}
We illustrate that, like the truncation error, the round-off error has a significant influence on the reliability of numerical simulations of chaotic dynamic systems. Due to the butterfly-effect, all numerical approaches in double precision cannot give a reliable simulation of chaotic dynamic systems. So, in order to avoid man-made uncertainty of numerical simulations of chaos, we had to greatly decrease both of the truncation and round-off error to a small enough level, plus a verification of solution reliability by means of an additional computation using even smaller truncation and round-off errors.
\end{abstract}

\section{Introduction}

The sensitive dependence on initial conditions (SDIC) was first found in 1890 by Henri Poincaré [1] in a particular case of the three-body problem, who later proposed that such phenomena could be common, say in meteorology [2]. In 1963, using a digit computer (Royal McBee LGP-30) to solve a set of coupled ordinary differential equations (ODEs)

$$
\left\{\begin{array}{l}
\dot{x}=-\sigma x+\sigma y, \\
\dot{y}=r x-y-x z, \\
\dot{z}=x y-b z,
\end{array}\right.
$$

where $\sigma, b$ and $0<r<+\infty$ are physical parameters, Edward N. Lorenz [3] found the so-called butterfly-effect: a tiny change in initial condition might result in large difference in a later state. The tiny difference in initial condition of such kind of chaotic dynamic systems enlarges exponentially [4], which can be characterized by a positive Lyapunov exponent $\lambda$. In other words, the maximum Lyapunov exponent of a chaotic dynamic system must be positive. However, Lorenz [5] also reported that, by means of the Runge-Kutta method with data in double precision, the maximum Lyapunov exponents of numerical simulations of a chaotic dynamic system given by different values of time-step may fluctuate around zero, say, its value constantly changes between positive and negative ones, even if the initial condition is exactly the same and the time step becomes rather small. Thus, the computer-generated numerical simulations of chaotic dynamic systems are sensitive not only to initial condition but also to numerical algorithms. This is easy to understand, since there always exist the truncation and roundoff errors at any steps of numerical simulations of chaos, 
which enlarge exponentially due to the so-called butterfly-effect. In addition, Teixeira et al. 6] investigated the time-step sensitivity of nonlinear atmospheric models and found that "different time steps may lead to different model climates and even different regimes", thus "for chaotic systems, numerical convergence cannot be guaranteed forever".

Hoover et al. [7] illustrated the Lyapunov's instability by comparing numerical simulations of a chaotic Hamiltonian system given by two Runge-Kutta and five symplectic integrators [8 10] in double precision, and found that "all numerical methods are susceptible to Lyapunov instability, which severely limits the maximum time for which chaotic solutions can be accurate", although "all of these integrators conserve energy almost perfectly" and "they also reverse back to the initial conditions even when their trajectories are inaccurate". As reported by Hoover et al. [7] , "the advantages of higher-order methods are lost rapidly for typical chaotic Hamiltonians", and "there is little distinction between the symplectic and the Runge-Kutta integrators for chaotic problems, because both types lose accuracy at the very same rate, determined by the maximum Lyapunov exponent."

Even for some dynamic systems without Lyapunov's instability, it is rather hard to gain accurate prediction, too. For example, let us consider the famous Lorenz equation (11) in the case of $\sigma=10, b=8 / 3$, which is chaotic only when $r \geq 24.74$. It is well-known that, when $1<r<24.74$, the long-term solution of the Lorenz equations should finally tend to one of the two stable fixed points

$$
C(\sqrt{b(r-1)}, \sqrt{b(r-1)}, r-1)
$$

and

$$
C^{\prime}(-\sqrt{b(r-1)},-\sqrt{b(r-1)}, r-1) .
$$

However, in the case of $r=22$, Li et al. [11] studied the sensitive dependance of the fixed point on the time-step $\Delta t$, which are calculated by means of many explicit/implicit numerical approaches (such as Euler's method, Runge-Kutta methods of orders from 2 to 6 , Taylor series methods of orders from 2 to 10, Adams methods of orders from 2 to 6 , and so on) in double precision, but found that the long-term results of numerical simulations are rather sensitive to the step size $\Delta t$, say, they always fluctuate between the two fixed points, no matter how small the time-step $\Delta t$ is. Thus, they made the conclusion that "numerical solution obtained by any stepsize is unrelated to exact solution" [11].

These numerical facts lead to some intense arguments. Some even believed that "all chaotic responses are simply numerical noise and have nothing to do with the solutions of differential equations" [12]. On the other side, using double precision data and a few examples based on the 15th-order Taylor-series procedure with decreasing time-step, Lorenz [13] was optimistic and believed that "numerical approximations can converge to a chaotic true solution throughout any finite range of time, although, if the range is large, confirming the convergence can be utterly impractical."

Is it possible to gain a convergent solution of a chaotic dynamic system in a long enough interval of time? This question is of critical importance. 
Note that convergent chaotic simulations cannot be guaranteed even if different high-order numerical methods were used, as illustrated by many researchers [6,7,11]. So, it is useless to reduce truncation errors only. Note that all of them used data in double precision, which leads to round-off error at each step, which also enlarges exponentially due to the butterfly-effect of chaos, just like truncation errors. So, to guarantee the convergence of chaotic solution, both of the truncation and round-off errors must be controlled to be much smaller than physical variables under investigation. In 2009, Liao [14] suggested the so-called "Clean Numerical Simulation" (CNS) [15,16] for chaotic dynamic systems and turbulence, which is based on the arbitrary order of Taylor expansion method [17, 18] and the use of all data in arbitrary precision (i.e. multiple precision [19]), plus a verification of solution reliability. By means of the CNS using the 3500th-order Taylor expansion method and data in 4180-digit precision, the convergent, reliable chaotic solutions of Lorenz equation were obtained even in $[0,10000]$, a rather long interval of time [20. Its solution reliability was further verified by means of the CNS using the 3600th-order Taylor series method and data in 4515-digit precision [20]. This work supports Lorenz's optimistic viewpoint that "numerical approximations can converge to a chaotic true solution throughout any finite range of time" [13].

Here, we further illustrate the effects of round-off error on the reliability of numerical simulations of chaotic dynamic systems, and show the importance of reducing both of truncation and round-off errors.

\section{Influence of round-off errors}

To investigate the influence of round-off error on the reliability of numerical simulations of chaotic dynamic systems, let us consider the Lorenz equation (1D) in the case of $\sigma=10, b=8 / 3$ and $r=23$, with the exact initial condition

$$
x(0)=5, y(0)=5, \quad z(0)=10 .
$$

Since its solution is chaotic when $r \geq 24.74$, the long-term numerical simulation should finally tend to one of the two stable fixed points in he case of $r=23$. Let $h=\Delta t$ denote the step-size. It is found that the final values of the numerical simulations given by the 4th-order Runge-Kutta method using data in double precision indeed rather sensitive to the step-size $h$, as shown in Fig. 1. No matter how small the stepzise $h$, the final values always fluctuate between the two fixed points. The same phenomenon was reported by Li et al. [11].

In order to reduce the truncation error, we use the $M$ th-order Taylor expansion method and the stepsize $h=0.01$ to gain numerical simulations of Lorenz equation (1) in the case of $\sigma=10, b=8 / 3$ and $r=23$ with the initial condition (2). Obviously, as $M$ becomes large, the truncation error could be rather small. To increase the computation efficiency, we use parallel computation by means of different numbers of processes, denoted by $n p$. 
(a)

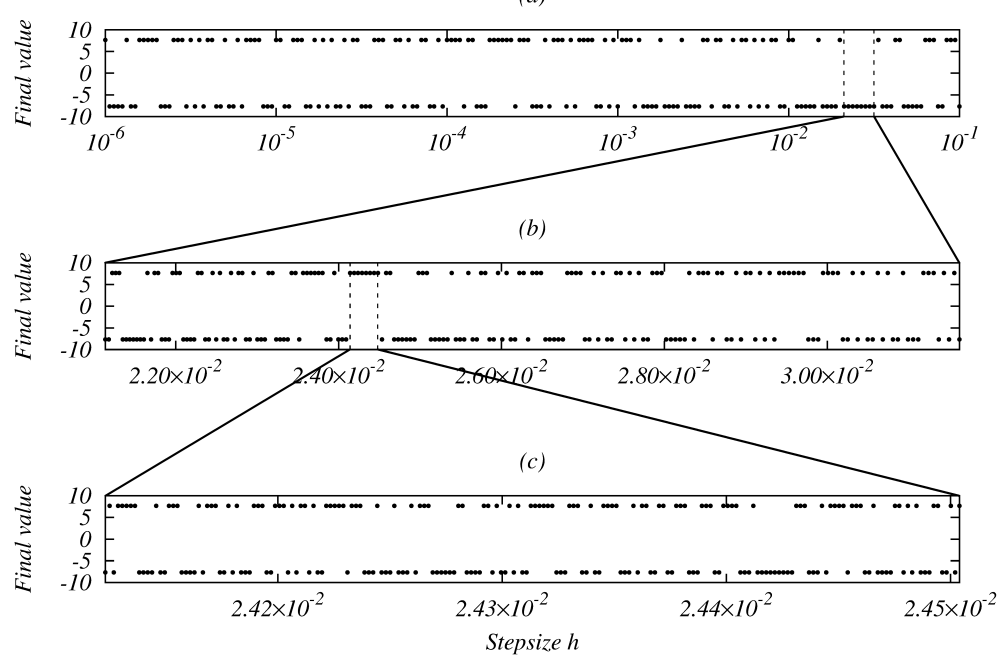

Figure 1: Final value of $x(t)$ of the Lorenz equations in case of $r=23$ with the initial value $(5,5,10)$ versus stepsize $h$, obtained by the 4 th-order Runge-Kutta method in double precision. The stepsize $h$ varies from (a) $10^{-6}$ to $10^{-1}$, (b) from $2.11349 \times 10^{-2}$ to $3.16228 \times 10^{-2}$ and (c) from $2.41107 \times 10^{-2}$ to $2.45086 \times 10^{-2}$, respectively.

It is found that, using the double precision, we gain different final values by means of high-order Taylor expansion method even using the same laptop (Thinkpad L440 with Intel Core i7-4712MQ) but different numbers of processes, as shown in Fig. 2. Even if the order of Taylor expansion method is rather high, such as $M=200$, corresponding to rather small truncation error, we still gain different final values using the same laptop but different numbers of processes, as shown in Fig. 3. It is found that, using the high-order Taylor series method in double precision, we would gain different final values using the same number of processes $n p$ but different computers, as shown in Fig. 4. This kind of man-made uncertainty of numerical simulations cannot be avoided even by means of rather high order of Taylor series method such as $M=200$, as shown in Fig. 5. All of these illustrate that decreasing truncation error alone cannot avoid the man-made uncertainty of numerical simulations for the considered problem.

As pointed out by Monniaux [21], even using the same programmes with the same compiler, which have exactly the same expression, the same values in the same variables and so on, different working platforms may exhibit subtle differences with respect to floating-point computations. Thus, both of the different number of processes operating on the same computer and the different computers with the same number of processes can generate rather tiny difference of round-off errors, which unfortunately would be enlarged so greatly (due to the butterfly-effect of chaos) that completely different numerical simulations might be obtained! In practice, round-off error sometimes indeed might lead to some serious problems, such as the system failure in the military: on February 25, 1991, a loss of significance in a MIM-104 Patriot 
missile battery prevented it from intercepting an incoming Scud missile in Dhahran, Saudi Arabia, contributing to the death of 28 soldiers from the U.S. Army's 14th Quartermaster Detachment [22].

Such kind of man-made uncertainty of numerical simulations of the Lorenz equation can be avoided by decreasing both of the truncation and round-off errors at the same time! It is found that, using the $M$ th-order Taylor series method $(M \geq 130)$ in the 512-digit precision, all numerical simulations agree quite well in the whole interval of time and besides tend to the same fixed point, even if different numbers of processes are used on different computers, as shown in Figs. 6-8. All of these examples illustrate the importance of decreasing both of truncation and round-off errors to the reliability of numerical simulations of chaotic dynamic systems, such as Lorenz equation, three-body problem and so on. Therefore, it clearly indicates that, using numerical approaches in double precision, one can not avoid the man-made uncertainty of numerical simulations for chaotic dynamic systems, as shown by many researchers [3, 5, 7, 11, 12].

The above-mentioned examples also explain why one had to use the 3500th-order Taylor expansion method in the 4180-digit precision [20] so as to gain a convergent numerical simulation of a chaotic solution of Lorenz equation in a rather long interval of time $[0,10000]$, whose reliability was further verified by means of the 3600th-order Taylor series method and data in 4515-digit precision. Nowadays, the importance of using multiple-precision data to gain reliable numerical simulations of chaotic dynamic systems receives recognition by more and more researchers [23 28].

\section{Concluding remarks and discussions}

We confirm that, due to the butterfly effect, the traditional numerical approaches in double precisions indeed cannot give reliable numerical simulations of chaotic dynamic systems. Thus, decreasing the truncation error alone cannot avoid the man-made uncertainty of numerical simulations of chaos. However, such kind of man-made uncertainty of numerical simulations for chaotic dynamic systems can be completely avoided by decreasing both of the truncation and round-off errors at the same time, plus a verification of solution reliability by means of additional computations using even smaller truncation and round-off errors.

In this paper we illustrate that, due to the butterfly-effect, even the very tiny difference of round-off error caused by different numbers of processes or different computers might lead to significant variation of numerical simulations of a chaotic dynamic system. So, the butterfly-effect is indeed a huge obstruction for us to gain reliable numerical simulations of chaos in a long interval of time. Note that a few current numerical investigations suggest that turbulent flows might be sensitive even to micro-level thermal fluctuation [28]. Then naturally, the turbulent flows should be also sensitive to numerical noises. Thus, it should be of benefit to study the influence of numerical noises to numerical simulations of turbulence 


\section{References}

[1] J. H. Poincaré, Sur le probléme des trois corps et les équations de la dynamique. Divergence des séries de m. Lindstedt, Acta Math. 13 (1890) 1-270.

[2] S. Wolfram, A New Kind of Science, Stephen Wolfram, LLC, 2002.

[3] E. N. Lorenz, Deterministic nonperiodic flow, Journal of the Atmospheric Sciences 20 (2) (1963) 130-141.

[4] J. C. Sprott, Elegant Chaos: Algebraically Simple Chaotic Flows, World Scientific, Singapore, 2010.

[5] E. N. Lorenz, Computational periodicity as observed in a simple system, Tellus-A 58 (2006) $549-559$.

[6] J. Teixeira, C. Reynolds, K. Judd, Time step sensitivity of nonlinear atmospheric models: Numerical convergence, truncation error growth, and ensemble design, J. Atmos. Sci. 64 (2007) $175-188$.

[7] W. Hoover, C. Hoover, Comparison of very smooth cell-model trajectories using five symplectic and two runge-kutta integrators, Computational Methods in Science and Technology 21 (2015) 109 - 116.

[8] H. Yoshida, Construction of higher order symplectic integrators, Physics Letters A 150 (5) (1990) 262-268.

[9] A. Farrés, J. Laskar, S. Blanes, F. Casas, J. Makazaga, A. Murua, High precision symplectic integrators for the solar system, Celestial Mechanics and Dynamical Astronomy 116 (2) (2013) 141-174.

[10] R. I. McLachlan, K. Modin, O. Verdier, Symplectic integrators for spin systems, Physical Review E 89 (6) (2014) 061301.

[11] J. Li, Q. Zeng, J. Chou, Computational uncertainty principle in nonlinear ordinary differential equations (i) numerical results, Science China Technological Sciences 44 (1) (2001) 55-74.

[12] L. Yao, D. Hughes, Comment on "computational periodicity as observed in a simple system" by Edward N. Lorenz (2006), Tellus-A 60 (2008) 803 - 805.

[13] E. N. Lorenz, Reply to comment by L.-S. Yao and D. Hughes, Tellus-A 60 (2008) $806-807$.

[14] S. Liao, On the reliability of computed chaotic solutions of non-linear differential equations, Tellus A 61 (4) (2009) 550-564.

[15] S. Liao, On the numerical simulation of propagation of micro-level inherent uncertainty for chaotic dynamic systems, Chaos, Solitons \& Fractals 47 (2013) 1-12. 
[16] S. Liao, Physical limit of prediction for chaotic motion of three-body problem, Communications in Nonlinear Science and Numerical Simulation 19 (3) (2014) 601-616.

[17] G. Corliss, Y. Chang, Solving ordinary differential equations using Taylor series, ACM Trans. Math. Software 8 (1982) 114-144.

[18] R. Barrio, F. Blesa, M. Lara, VSVO formulation of the Taylor method for the numerical solution of ODEs, Computers \& Mathematics with Applications 50 (1) (2005) 93-111.

[19] P. Oyanarte, MP-a multiple precision package, Computer Physics Communications 59 (2) (1990) 345-358.

[20] S. Liao, P. Wang, On the mathematically reliable long-term simulation of chaotic solutions of lorenz equation in the interval [0,10000], Science China - Physics, Mechanics \& Astronomy 57 (2) (2014) 330-335.

[21] D. Monniaux, The pitfalls of verifying floating-point computations, Acm Transactions on Programming Languages and Systems 30 (3) (2008) 1-41.

[22] Office of U. S. Government Accountability, Patriot Missile Defense: Software Problem Led to System Failure at Dhahran, Saudi Arabia, Technical Report GAO/IMTEC-92-26 (1992).

[23] B. Kehlet, A. Logg, A reference solution for the Lorenz system on [0, 1000], in: AIP Conference Proceedings, Vol. 1281, 2010, p. 1635, (doi: 10.1063/1.3498141).

[24] S. A. Sarra, C. Meador, On the numerical solution of chaotic dynamical systems using extend precision floating point arithmetic and very high order numerical methods, Nonlinear Analysis: Modelling and Control 16 (2011) 340 - 352.

[25] P. Wang, J. P. Li, Q. Li, Computational uncertainty and the application of a high-performance multiple precision scheme to obtaining the correct reference solution of lorenz equations, Numer Algorithms 59 (2012) 147 - 159.

[26] P. Wang, Forward period analysis method of the periodic hamiltonian system, PLoS ONE 11 (2016) e0163303.

[27] R. Barrio, A. Denab, W. Tucker, A database of rigorous and high-precision periodic orbits of the lorenz model, Computer Physics Communications 194 (2015) $76-83$.

[28] Z. Lin, L. Wang, S. Liao, On the origin of intrinsic randomness of RayleighBénard turbulence, Sci. China - Phys. Mech. Astron. 60 (1) (2017) 14712. 


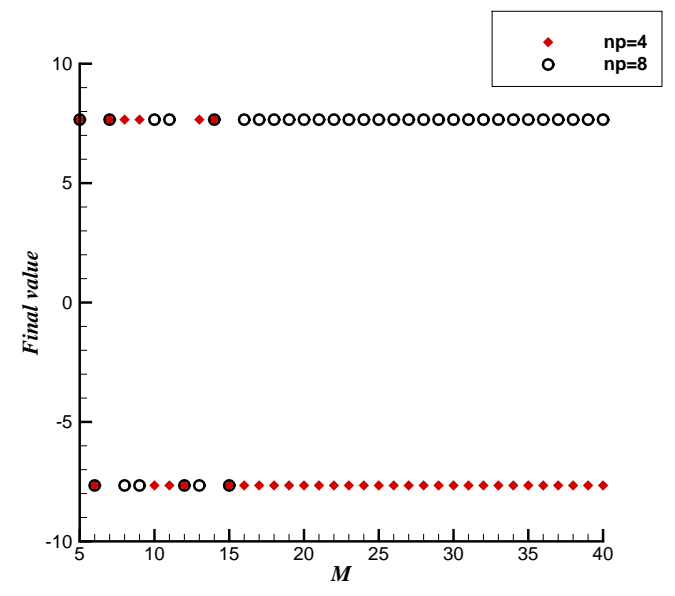

Figure 2: Final values of numerical simulation of $x(t)$ of the Lorenz equations in case of $r=23$ with the initial value $(5,5,10)$ versus $M$ (i.e. the truncated $M$ thorder Taylor's expansion method), given by the same laptop (Thinkpad L440 with Intel Core i7-4712MQ) using data in double-precision but the different $n p$ (number of processes).

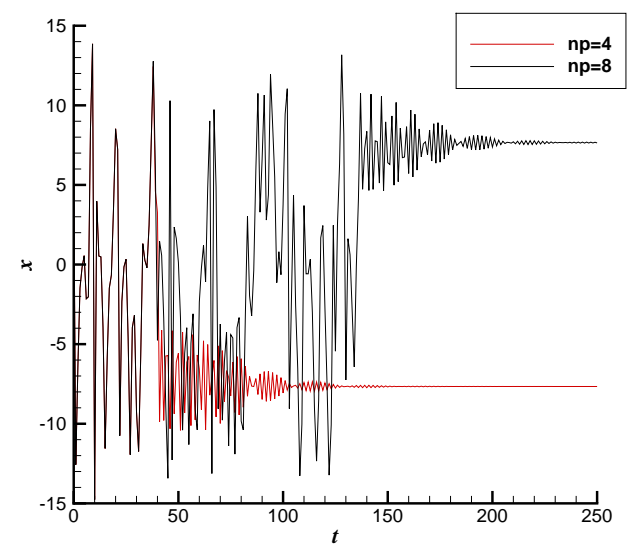

Figure 3: The numerical simulations of $x(t)$ of the Lorenz equations in case of $r=23$ with the initial value $(5,5,10)$, given by the same laptop (Thinkpad L440 with Intel Core i7-4712MQ) using the 200th-order Taylor' expansion method (i.e. $M=200$ ) and data in double precision but the different $n p$ (number of processes). 


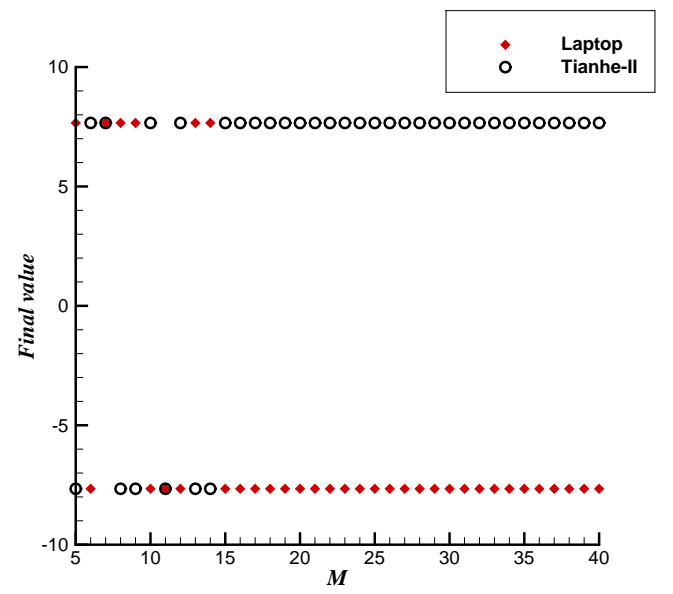

Figure 4: Final value of numerical simulations of $x(t)$ versus $M$ (i.e., the truncated $M$ th-order Taylor's expansion), given by means of the same number of processes $(n p=4)$ and data in double precision but the different computers, i.e. the laptop (Thinkpad L440 with Intel Core i7-4712MQ) and the supercomputer Tianhe-II, respectively.

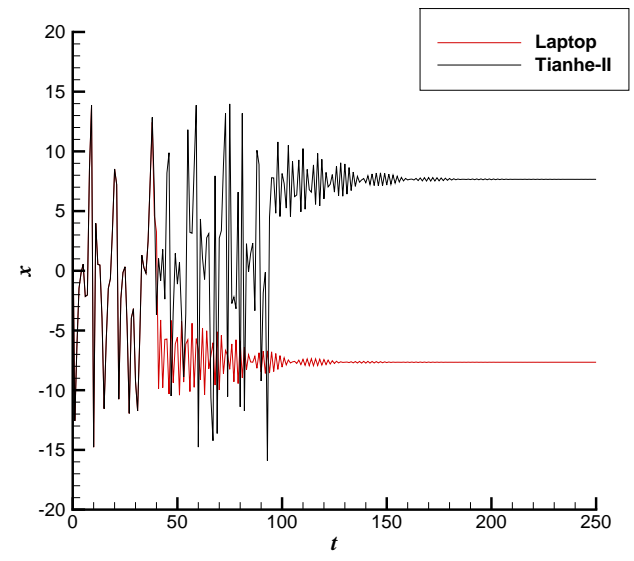

Figure 5: The numerical simulations of $x(t)$, given by the 200th-order Taylor's expansion method (i.e. $M=200)$ using the same number of processes $(n p=4)$ and data in double precision but the different computers, i.e. the laptop (Thinkpad L440 with Intel Core i7-4712MQ) and the supercomputer Tianhe-II, respectively. 


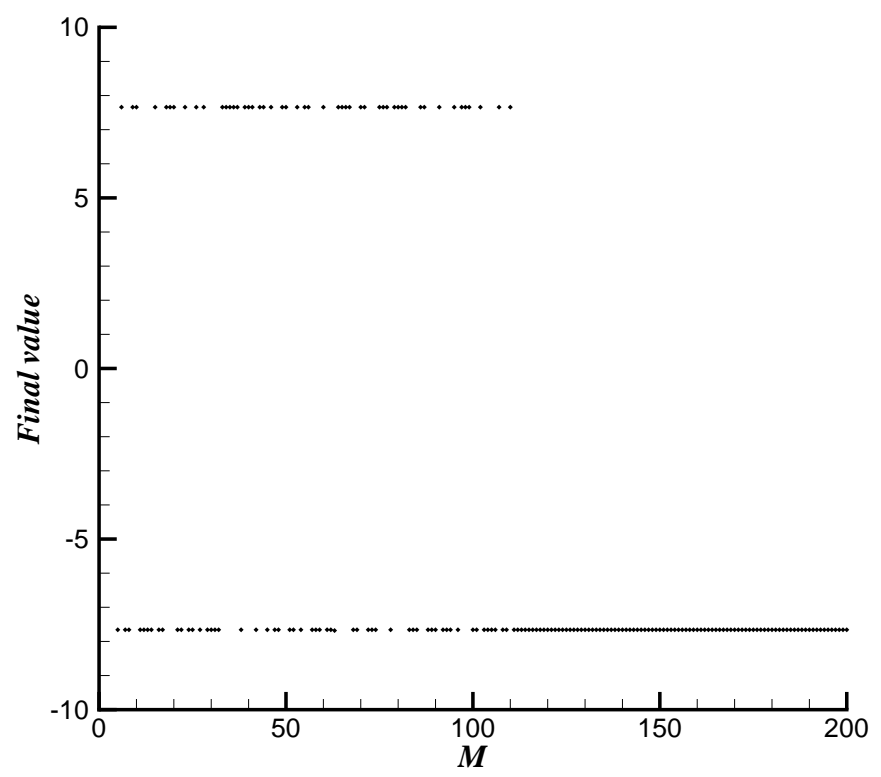

Figure 6: Final value of the numerical simulation of $x(t)$ of the Lorenz equations in case of $r=23$ with the initial value $(5,5,10)$ given by means of the $M$ th-order Taylor's expansion using data in 512-digit precision. For all given $M$, the same final values of $x(t)$ are obtained, which are independent of the computers (i.e. the laptop and the supercomputer) and the number of precesses $(n p=4$ or 8 ). As $M \geq 130$, the final value becomes to be independent of $M$, too.

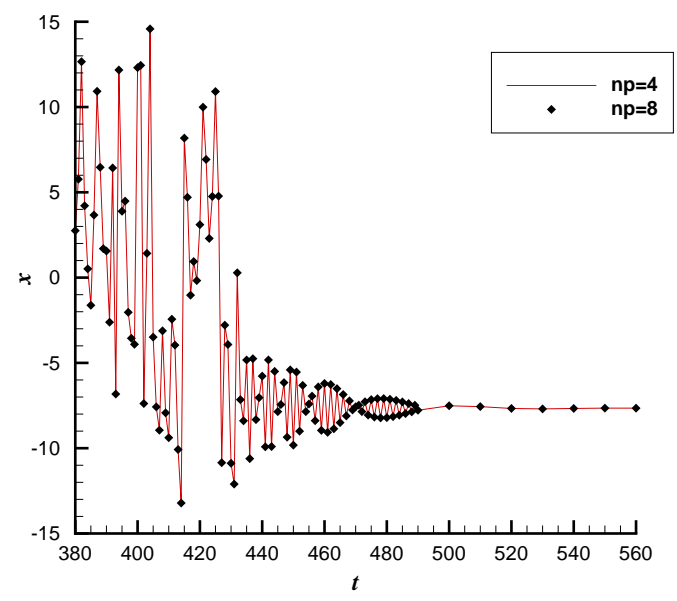

Figure 7: The numerical simulations of $x(t)$ given by the 200th-order Taylor's expansion method (i.e. $M=200$ ) and data in 512-digit precision using the same laptop (Thinkpad L440 with Intel Core i7-4712MQ) but different $n p$ (number of processes). 


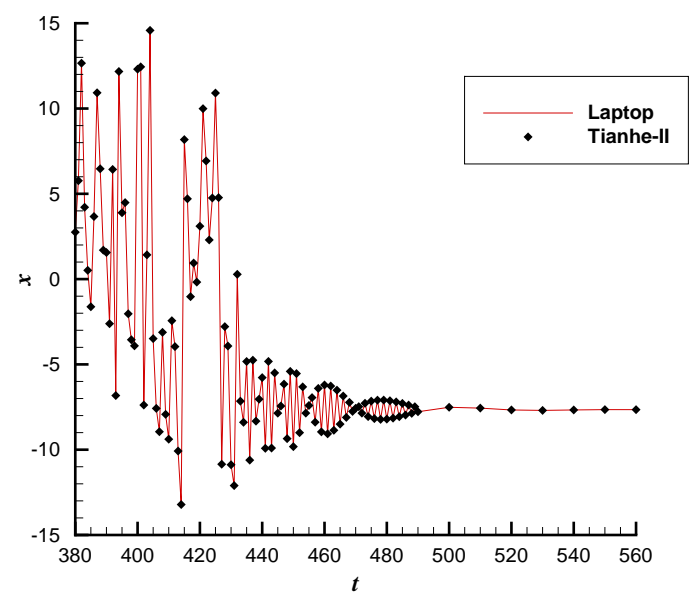

Figure 8: The numerical simulations of $x(t)$ given by means of the 200th-order Taylor's expansion method (i.e. $M=200$ ) and data in 512-digit precision using the same number of processes $(n p=4)$ but the different computers, i.e. the laptop (Thinkpad L440 with Intel Core i7-4712MQ) and the supercomputer Tianhe-II, respectively. 